\title{
Respiratory Infections: Community-Acquired Pneumonia and Newer Microbes
}

\author{
H. Y. Reynolds
}

Milton S. Hershey Medical Center, The Pennsylvania State University, Hershey, Pennsylvania 17033

\begin{abstract}
Respiratory infections, especially community-acquired forms of pneumonia (CAP), are challenging for clinicians because (1) a causative microorganism can only be found in about $50 \%$ of cases; (2) initial therapy, therefore, must be based on a probable or most likely etiology in the context of the patient's overall medical condition; and (3) new microbes or those considered previously as normal flora or less virulent forms seem responsible for some cases. It is important to be acquainted with new causes of infection which include Legionella species, Chlamydia pneumoniae, diphtheroids in certain instances (Corynebacterium pseudodiphtheriticum), and viruses such as the Hanta strains. Infections with Bordetella pertussis are increasing. However, the ever present and most common cause of CAP, Streptococcus pneumoniae, continues to present problems because of increasing antibiotic resistance, the high case fatality rate when bacteremia accompanies pneumonia, and the inability to give prophylactic immunization to all people with risk factors for this infection.
\end{abstract}

Key words: Respiratory infection-Pneumonia

\section{Introduction}

Respiratory infections are among the most common afflictions of healthy people, and their treatment plus the associated inconvenience, morbidity and mortality [78], and newer microbial causes that are being encountered, make this illness important and ever changing [27]. As community-acquired forms of respiratory infection are most common [81], this review will concentrate on community-acquired pneumonia (CAP) and the principal organisms involved. However, this review will not include CAP in immunocompromised patients (recently investigated by Mundy and colleagues [61]), thus excluding respiratory infections and tuberculosis in HIV-infected subjects, and it 
does not cover nosocomial pneumonia in patients receiving assisted ventilation in an intensive care unit.

\section{Pneumonia, Community Style}

The importance of pneumonia, recently defined by Marrie [54] as a condition of inflammation and consolidation of lung tissue caused by an infectious agent, is considerable; it remains a leading cause of death and morbidity and consumes a large amount of patient health care costs. Pneumonia occurs frequently, but the attack rate varies greatly by age, from associated comorbid conditions and among people with different ethnic and sociologic backgrounds. CAP has witnessed new developments that will be the focus of this review: (1) frequency with which an actual microbial diagnosis is established; (2) recent guidelines to help with initiating empirical treatment based on the probable presence of pathogens; and (3) new causative microbes. Not all of these latter organisms are associated with pneumonia but do cause respiratory infection.

First, the most vexing problem is that the etiology of so many cases of CAP remains elusive. When three series of patients with CAP that required hospitalization $[10,24,55]$ were tabulated and nicely compared by Marrie (see Table 4 in Ref. 54) according to microbial causes, the number (percentage) of patients in whom the etiology of pneumonia remained unknown was 33\% [24], 47\% [55], and approximately $49 \%$ [10]. To elaborate from one study [10] that conscientiously sought the etiologic organism(s) in acute pneumonia, this was only established in approximately $50 \%$ of cases. For 198 patients with 204 episodes of pneumonia investigated and given followup at the McClellan Memorial Veterans Medical Center (Little Rock, Arkansas), a specific agent was found or attributed in 103 episodes; for 154 episodes of CAP, a diagnosis was made in 79 cases $(51 \%)$ and in 24 of 50 patients (48\%) with hospitalacquired pneumonia. Because these patients had complete and long term follow-up, some diagnoses were substantiated by serial serologic titers, an approach not always possible. Most patients $(>90 \%$ ) had an important coexistent disease process, such as malignancy, chronic obstructive pulmonary disease (COPD), or heart failure [10], which would be expected in an elderly patient group (mean age 64 years) of military veterans.

However, as mentioned by Marrie [54], there are always several problems in finding the causative organism in CAP. (1) Many of the patients are unable to produce spontaneously a good sputum specimen for analysis and culture (as an example, 23\% of patients did not have purulent sputum in the Bates et al. series of patients [10]. (2) Many patients have been started on antibiotic treatment before presentation (this situation occurred for $28 \%$ of CAP patients and $32 \%$ of patients with hospital-acquired pneumonia in the Bates et al. series [10]). (3) Some patients will have an infective agent that cannot be cultured easily from sputum or may require special culture media [54].

Undoubtedly, the fact that a plausible causative microbe can be found at best in only about half of cases $[10,24,52,54,55]$ was one stimulus for a consensus approach to establish guidelines and a framework for initial management and treatment of adults with CAP [72] which, in the final analysis, concluded that antibiotic therapy often 
should be given empirically [72]. This is a summary statement from a distinguished panel that evaluated current epidemiology and trends in therapy and had a realistic appreciation of evolving health care practices. Therefore, these recommendations, endorsed as an official statement by the American Thoracic Society, for the initial management of CAP are important and merit a careful in-depth review [72]. Another recent discussion of CAP also emphasizes many of these points [9].

A focus of this statement was to determine how serious do the patient and the illness seem to be so that an initial decision can be made whether to treat as an outpatient or hospitalize the patient. Essential information to factor into the decision includes the patient's age; comorbid illnesses that may affect outcome or change the usual mixture of microbial flora colonizing the patient's nasooropharynx; the person's support system (available care giver) and living arrangements; and objective physical findings, vital signs, and screening laboratory tests at initial examination.

Based on the above, but always including the clinician's intuition about the patient's condition, a good decision can be made whether to initiate home care treatment, with family/or a nurse/home health care team, or to hospitalize. This is predicted on the likelihood that one or more of the usual microbial pathogens associated with CAP will be the etiology of the pneumonia [72]. Most commonly the microbes will be Streptococcus pneumoniae, respiratory viruses, Mycoplasma pneumoniae, Hemophilus influenzae, Legionella species (L. pneumophila most likely), Chlamydia pneumoniae, and perhaps Staphylococcus aureus. This spectrum might change, if the patient has COPD/ bronchitis, to enhance the probability of $S$. pneumoniae or Hemophilus sp. infection even more but also would make inclusion of Moraxella catarrhalis and Corynebacterium pseudodiphtheriticum (as will be discussed later) necessary. If the patient is older and/or frail and debilitated, Gram-negative enteric pathogens may more likely be involved. The spectrum of microbes and the dynamics of their colonization of the respiratory tract are fascinating and complex and an interesting topic of its own [51, $71,80]$.

Other coexisting medical problems such as renal or liver insufficiency, diabetes mellitus, therapy causing immunosuppression, or cancer would enhance the etiologic possibility of an aerobic Gram-negative bacillus as carried in the colon or colonizing the skin (Pseudomonas aeruginosa). Aspiration of oropharyngeal secretions that can introduce anaerobic flora is a concern as well. Current epidemiologic information about infections in the local community, the immunization status of the patient (prior influenza and pneumococcal vaccine prophylaxis), and recent antibiotic usage are important. Any of these conditions might change a microbe from a "usual pathogen" to a more invasive and opportunistic one, making hospitalization necessary because of the need for parenteral antibiotics and monitoring of renal function. If, however, infection with a usual pathogen is probable, oral administration of a macrolide or of a secondgeneration cephalosporin (cefuroxime) is usually sufficient [72] in the absence of the extenuating factors just discussed. Thereafter, the patient's response to therapy over the next 2- to 3-day interval will dictate how to proceed. If there is a good response, the treatment is continued through completion, usually a 10-day antibiotic course. If not, a poor patient response may require a change or an addition of another antibiotic, or hospitalization could be deemed necessary to give additional supportive care, supplemental oxygen, or more intensive respiratory therapy. 
Noticeable in this approach is the absence of reliance on traditional diagnostic studies, even foregoing a standard chest radiograph as being desirable but not "practical in all situations" [72]. Hopefully, stringent cost considerations in managed care practice will not dictate this situation, for finding coexisting conditions, aptly mentioned by the committee [72], can be helpful, such as a pleural effusion or mass lesion causing bronchial obstruction. The other tests deemphasized are Gram stain analysis of sputum and routine aerobic bacterial cultures because sensitivity and specificity are low, and concordance between the Gram stain and subsequent culture results, S. pneumoniae for example, is poor. The futility of this analysis was supported in the Bates et al. [10] report, wherein viewing a single organism on the Gram stain of a good, suitable sputum specimen was correlated with the correct pathogen in only a very few cases. The usefulness of these tests is discussed in depth by Bartlett and Mundy [9]. Moreover, invasive procedures to obtain lower airway specimens not contaminated by oropharyngeal flora, such as bronchoscopy with a protected brush catheter, are not indicated for most patients with CAP; an exception of course is the seriously ill patient. Blood cultures are recommended for a hospitalized patient with CAP. However, if circumstances permit in a clinic or medical office setting, two blood cultures might be a good investment; since pneumococcal pneumonia is such a common cause of CAP, blood cultures can be positive in $20-30 \%$ of cases, and the case fatality rate is still appreciable. Admittedly, outpatient-obtained blood cultures present issues of quality control and require appropriate handling of the specimens for optimal recovery of microbes, but the yield can be helpful and high.

In summary, CAP is encountered frequently in all medical practice situations, and decisive and effective therapy must be designed and provided for these patients. Thus, the guidelines as presented [72] are a helpful attempt to devise clinical pathways to handle the illness expeditiously. As the recommendations will not satisfy every situation, their applicability, as discussed by Fein and Niederman [26], needs to be critiqued as new information is obtained or personal physician usage of them dictates. In addition, for the future, new techniques must be perfected which more rapidly detect the organisms or their products known to cause infection and identify the still unrecognized or additional infectious agents, as postulated by Bates and colleagues [10], which may account for some of the other $50 \%$ cases of CAP that do not yet yield a diagnosis using the best conventional methods to date. For example, the measurement of plasma cytokines [77] has the potential to gauge the host's response to infection and help with better determining how ill the patient is.

\section{Specific Infections}

\section{Streptococcus pneumoniae}

Pneumococcal infection is the most common cause of bacterial CAP and accounts for a widely variable percentage of the cases, ranging from 15 to $76 \%$ in representative patient series presented by Musher [63]. Mandell [52] cites several studies that report an incidence rate varying from 8 to $46 \%$, and he makes the point that this variation may 
reflect prior taking of antibiotics, obtaining appropriate sputum specimens, or use of specific antigen testing. Based on clinical responsiveness to penicillin therapy, it is likely that other cases considered to be of unknown microbial cause are in reality from S. pneumoniae [54]. This bacterium is often part of the usual microbial flora of the nasooropharynx of normals [51] but also seems to have a predilection for the airway mucosal surfaces of subjects with chronic lung diseases (as do Hemophilus species) [80]. Pneumococci can be aspirated in oropharyngeal secretions into the lower airways, whereupon they can attach to alveolar type II cells that create part of the epithelial lining surface of the alveolar spaces [98]. From a position on the surface of type II pneumocytes, the pneumococcal inoculum may either (1) be coated by nonimmune and/or immune opsonins present in the alveolar epithelial lining fluid and then phagocytosed by alveolar macrophages and polymorphonuclear (PMN) leukocytes which can intracellularly kill and remove them; or (2) microbial multiplication occurs, whereupon the pneumococcal capsule and cell wall components can stimulate local cytokines that alter permeability through or around alveolar type I epithelial cells, allowing (serum) fluid to enter the alveolar space. This provides a source of nutrients to sustain proliferation. Thereafter, the battle between host defenses and bacteria [13] ensues with the classical evolution of stages of lung consolidation occurring. The process has been updated nicely with new pathogenic insights and the array of cellular mediators [98].

One suspects [82] that the die is cast early on whether or not the host will develop clinical pneumonia and illness when a few wayward pneumococci detached in nasooropharyngeal secretions are aspirated into the lower airways. If the host is impaired for any of many reasons, i.e. an inebriated person who has a poor gag reflex and depressed cough response, one with hypogammaglobulinemia and inadequate IgG antibody levels, mucociliary clearance and cough reflex do not perform well, or IgG subclass $2\left(\mathrm{IgG}_{2}\right)$-specific antibody is not available, the pneumococci may gain a toehold. The failure to immunize with pneumococcal vaccine people who have significant risk factors is an important issue. Moreover, if the pneumococci represent one of the more virulent serotypes or the inoculum is large, these bacteria may begin with an advantage over the host. Pneumonia is likely.

Although pneumococcal infection is usually suspected and covered in the antibiotic spectrum used to treat a typical patient with CAP [72], successful management of this infection still presents important medical problems. These relate to: some difficulty in culturing these bacteria in sputum samples, if drying or microbial overgrowth of the specimen occurs; pneumonia with bacteremia remaining deadly with a case fatality rate of at least $20 \%$ despite appropriate antibiotic usage; increasing antibiotic resistance of $S$. pneumoniae to penicillin; and an administering of a reasonably protective and effective vaccine only to perhaps $25 \%$ of the adults who have indications to receive it. A few comments about each problem seem warranted.

Much has been written about obtaining a good specimen of sputum with minimal contamination of oropharyngeal fluid (saliva) for diagnostic microbiology and improving the correlation between Gram stain analysis and subsequent culture results. Treating an air-dried sputum smear with an omiserum antibody reagent for a capsular precipitin reaction (Quellung) to identify pneumococci more accurately and, therefore, improve upon Gram stain results was advocated in the past [58]. As current realism debates the usefulness of a sputum Gram stain to determine the cause of CAP $[9,10$, 
72], perhaps new molecular probe techniques will eventually do a better job [66, 88, $89]$ !

Invasive pneumococcal pneumonia with bacteremia has an attendant mortality in the $20 \%$ range; this is still surprising and difficult to accept, but deaths from this illness are not uncommon. Moreover, this basic fatality rate has not been reduced significantly in the past 30-year interval that has witnessed marvelous improvements in health care including many, many new antibiotics. Drs. Austrian and Gold [7] reported for 455 patients with pneumococcal pneumonia and bacteremia that 89 deaths resulted or 19.5\% fatal cases (from Table 1 in Ref. 7). For infection with serotype III, 18/35 (51\%) patients died. They found [7] that the incidence of bacteremia was 529 in 2,000 cases of pneumococcal pneumonia (26.5\%). Since then at least a dozen other published articles have reported case fatality rates for bacteremic pneumococcal pneumonia ranging from 21 to $41 \%$. A recent report by Örtqvist et al. [73] found this rate to be $28 / 107$ cases or $26 \%$. This continuing lack of success with this lethal form of infection still requires combined strategies of vigorous prophylaxis or prevention and better therapy.

Antibiotic resistance of $S$. pneumoniae to penicillin and to multiple antibiotics is of great concern, but whether this is a contributing factor in the persisting high case fatality rate in bacteremic pneumococcal pneumonia is uncertain. Administering higher doses of penicillin to compensate or using vancomycin usually provides adequate coverage. Pneumococcus with increased resistance to penicillin was isolated about 25 years ago [42]; other isolates from many parts of the world have been found with penicillin resistance $[5,28,46]$. The precise percentage of pneumococcal strains exhibiting penicillin resistance is difficult to compare among institutions and must be determined for each medical center, according to Dr. Appelbaum, ${ }^{1}$ who was one of the first to recognize this resistance [6]. For example, at the Pennsylvania State University's Milton S. Hershey Medical Center, he found that about 5-6\% of isolates recovered from blood and body fluids show resistance. Most of these isolates are from pediatric age patients. Recently, Hofmann and colleagues [43] found that from invasive pneumococcal infection, $25 \%$ of isolates were penicillin resistant in the Atlanta area.

Awareness of antibiotic resistance is changing the approach to treatment [30], especially by prescribing vancomycin or a third-generation cephalosporin. Another approach, proposed by Munford and Murphy [62], is to exploit the use of more immunogenic pneumococcal polysaccharide-protein-conjugated vaccines (currently being evaluated) and immunize children (over the age of 2 years) in daycare centers in an attempt to decrease their prevalence of the pneumococcal carrier state. These carriers often pass resistant strains to other children and adults.

If immunization strategies might reduce the respiratory tract carriage and spread of penicillin-resistant strains [62], then this approach certainly is applicable for preventing colonization to begin with and ensuring that a susceptible person also has circulating serum antipneumococcal antibodies, two effective methods for treatment. For prevention, the use of pneumococcal cell vaccines date to the early years of this century. In

'Personal comments from Peter C. Appelbaum, M.D., Ph.D., Professor of Pathology, Milton S. Hershey Medical Center, The Pennsylvania State University. 
the 1930-1940s vaccines containing pneumococcal capsular polysaccharide antigens were found to convey protection; moreover, the development of antibiotic resistance plus continuing mortality from bacteremic infection renewed interest in this form of therapy [6]. The polyvalent 14 serotype vaccine was licensed in the United States in 1977 and recommendations for its use given [2]. In 1983 the original vaccine was replaced by a vaccine containing capsular polysaccharides of 23 serotypes that would account for approximately $90 \%$ of bacteremic infections [92].

What is difficult to understand is why there has not been more successful advocacy by medical personnel to use this form of prophylaxis widely. Several critical studies have analyzed the clinical efficacy and cost effectiveness of the vaccine [representative reports $12,14,31,90,92,100]$, and all reports found it to have clinical utility. As an example [31], among 762 vaccine recipient patients (data from Minnesota Blue Cross and Blue Shield Insurance Plan), the risk of pneumonia was reduced after vaccination by about one third, or the vaccine provided an overall efficacy of $69 \%$. This varied from 50 to $75 \%$ among various risk categories, and the vaccine was more effective in women than men [31]. The total cost of vaccination was about $\$ 18.50$ per person [31]. Butler and colleagues [14], analyzing results from the Centers for Disease Control and Prevention's National Pneumococcal Surveillance Study (14 years in progress), found that overall efficacy was $57 \%$ for preventing infection caused by serotypes included in the pneumococcal vaccine, although this beneficial effect was not as good for certain patients with alcoholism or cirrhosis, sickle cell disease, hematologic malignancies, and chronic renal failure. For immunocompetent persons over the age of 65 years, efficacy was $70 \%$ [14]. Uniform efficacy has not always been found, however, for some higher risk subjects [93]. Thus, the vaccine does provide more protection in younger, immunocompetent subjects, but it is effective, albeit less so, in older people with associated illness.

On balance, this immunization therapy seems indicated [63] as reasonably effective preventive therapy for many people who have risk factors for this infection. The unfortunate situation is that so few who need the vaccine actually receive it. Perhaps one third of persons aged 50-64 years has a medical risk condition that would warrant the vaccine, but less than $10 \%$ receive it; among those over 64 years of age, one half has an indication to receive the vaccine, but only $15 \%$ or so receives it $[3,96]$. It is incumbent upon medical practitioners to insist that our patients receive this vaccine, if indicated. In a similar vein, evidence supports that influenza vaccination reduces the risk of clinical influenza in the elderly or other persons with indications to receive it. A recent metaanalysis of 20 cohort studies, performed by Gross and colleagues [39], emphasized this conclusion. Sadly, half of the elderly people do not [68], and we must work to improve this situation $[35,60,68]$.

Finally, the question of administering preformed specific antibody for serious pneumococcal pneumonia should be reassessed. If vaccination to induce antibody is beneficial in preventing or improving the outcome from the illness, then passive immunity in the form of IgG antibodies [84] should have a role. Certainly, the continued high case fatality rate from bacteremic disease begs for new or more aggressive therapeutic approaches. Commercially available immunoglobulin preparations, assayed by Hamill and colleagues [40], were found to contain IgG-reactive specific antibody against several common serotypes of $S$. pneumoniae (serotypes 3, 4, 8, 14, 
and $19 \mathrm{~F}$ ); the titers were variable, however, among the four commercially available IVIG products compared [40]. This antibody was a likely result of a humoral immune response among certain globulin donors who had experienced prior pneumococcal infection, or possibly some donors were vaccinated. This human antipneumococcal antibody was found also to be protective in a mouse infection assay [40]. As drugresistant organisms increase, and $S$. pneumoniae is a pertinent example of emerging penicillin resistance, many health care scientists warn that we are entering a postantibiotic era, thus antibody-based therapies may reemerge [17].

\section{Legionella Infection}

The realization that for about one half the cases of CAP no specific microbial cause can be documented $[10,54,72]$ has certainly whetted the imagination that as yet undiscovered microbes or commensal oropharyngeal flora with unsuspected pathogenicity in the lower airways will be found. Perhaps no bacterium in recent experience has exemplified this expectation of a "new find" more than the Legionella species. The July 1976 epidemic in Philadelphia publicized pneumonia occurring among State Legionnaires at a convention in an unforgettable way and raised the specter of a new killer respiratory infection $[29,57,79]$. This was the first in a series of spectacular, exotic respiratory epidemics that have caused apprehension among the American public; the emergence of drug-resistant tuberculosis and the viruses Ebola and Hanta have continued the pattern. Perhaps even another infection, similar to but not hemorrhagic dengue fever, is occurring in Nicaragua, where an epidemic is developing. This febrile illness can cause pulmonary hemorrhage and suffocation [75].

Legionella pneumonia is a serious and fatal infection, as recorded in the first epidemic in which $16 \%$ (29/182 patients) died; preexisting illness was a factor in many cases [29]. Therefore, the host who successfully withstands the infection may have special resistance or can develop it quickly. The incubation period for the disease ranges from 2 to 10 days, although low grade intermittent exposure can occur.

Quickly, it was established that Legionella infection was not entirely new but had occurred before and in fact solved the mystery of some unexplained outbreaks in the past. Moreover, a flu-like, nonpneumonic respiratory illness (Pontiac fever) was part of the spectrum as well [33], and had occurred in 1968. Soon, other species of Legionella were identified; the Pittsburgh pneumonia agent [65] later became Legionella micdadei [25]. Recently, a review by Skerrett [94] indicated that 40 species of the genus Legionella have been identified and more than 50 serotypes found. About half of these have been associated with pneumonia, but $L$. pneumophila still accounts for the majority of infections $(80 \%)$. Two reviews $[22,85]$ comprehensively cover all of the features of these small aerobic Gram-negative bacteria and the infections caused.

Infections with Legionella sp. are fascinating for several reasons: (1) the multiple settings in which they crop up; (2) the adaptive mechanisms required by the host to resist this potentially deadly intracellular bacterium ( $L$. pneumophila has been studied most intensively); and (3) the therapeutic potential for local immunomodulatory airway therapy. Legionella is estimated to cause $1-15 \%$ of the cases of CAP, but its prevalence has seemed to vary geographically [22] and among neighboring medical centers. For 
the three series of CAP requiring hospitalization, tabulated and contrasted for etiology by Dr. Marrie [Table 4 in Ref. 54] Legionella species accounted for 6.7\% [24], 2.2\% [55], and 9\% [10] of CAP cases. Legionella sp. have been isolated infrequently by special cultures from patient specimens at our medical center in Hershey, Pennsylvania. In fact, only one positive culture has been obtained in the past 2 years, despite about 10-12 sputum or respiratory specimens being evaluated per month. ${ }^{2}$ However, about 60 miles away in Chambersburg, Pennsylvania, near the Pennsylvania-Maryland state border, the community's hospital was the center of a publicized epidemic this past summer [18, 47]. At least 35 cases of Legionella infection occurred in July 1995 among personnel who were exposed to an aerosol generated by an external water source. Experiencing a nearby epidemic heightened awareness of this infection in our general area.

A point or common source outbreak is usually associated with contaminated water in community-acquired or nosocomial cases of Legionella infection. The usual circumstances or environmental conditions reported [59] involve a warm water reservoir, perhaps suboptimal concentrations of chlorine as a disinfectant, concomitant growth of other organisms (algae) providing nutrients, and an aerosol or droplet-generating mechanism. Presumably, direct droplet inhalation or microaspiration of oropharyngeal secretions containing the organisms is the route that inoculates the lung. Transmission of Legionella causing sporadic cases of nosocomial pneumonia may occur in obscure ways, humdifiers or rinsing ventilatory equipment with tap water, as was demonstrated experimentally [102]. Although there are many unsuspecting ways this bacterium might be presented to a patient, person-to-person transmission does not occur [85]. Epidemic occurrences may have similarities in clinical presentation, and sporadic cases can occur year round, especially in immunocompromised and organ transplant recipient patients. This possibility may not be entertained in the differential diagnosis of pneumonia. Recent reviews $[22,85]$ of this infection continue to stress that no constellation of patient symptoms, signs, or screening laboratory tests is sufficiently pathognomonic or reliably distinct to permit a confident clinical diagnosis of Legionella infection.

A special culture medium method of detecting antigenuria through urine testing is more sensitive and now preferable to the direct immunofluorescent antibody method. However, use of several screening tests, including sputum culture, serum antibody titer, and urinary antigen, is recommended by Plouffe and Ingram [45, 74] to determine conclusively that Legionella infection is present or excluded. A general concern is that Legionella pneumonia is perhaps underestimated as a cause of CAP, especially in the setting of severe CAP.

Host defense mechanisms have been studied extensively in Legionella infections, and fascinating insights are known [11, 16, 44, 67, 95]. The humoral antibodies elicited against Legionella antigens, when used experimentally to opsonize L. pneumophila bacteria and complement is added, do not cause lysis of the bacteria [44]; instead they are phagocytosed by monocytes and alveolar macrophages where intracellular prolif-

\footnotetext{
${ }^{2}$ Personal comments from Suzanne Schick, Chief Technologist, Microbiology Laboratory, Milton S. Hershey Medical Center, The Pennsylvania State University.
} 
eration of the bacteria occurs with ultimate destruction of these phagocytic cells [67]. If the alveolar macrophages can be "activated" by cytokines, inhibition of $L$. pneumophila multiplication occurs [67]. This activation process was found to be interferon gamma (IFN- $\gamma$ ) [11], one of several potent cytokines that stimulate macrophages (this topic is reviewed in Ref. 83). INF- $\gamma$ was able to down-regulate cell surface receptors for transferrin, thus decreasing the amount of iron transported into the cell [16]. An essential nutrient for the bacterium's intracellular growth may be diminished so that multiplication and growth cannot be sustained [16]. In an animal model, administration of this cytokine into the airways facilitated lung defense against the organisms [95].

The potential usefulness of immunomodulatory therapy for Legionella lung infection follows from the experimental studies cited above $[16,95]$ and may be translated into clinical research. As INF- $\gamma$ has been safely aerosolized into human airways [56], $\mathrm{INF}-\boldsymbol{\gamma}$ administered into the lungs could have a therapeutic role in Legionella pneumonia as found in the animal model [95]. This might become adjunctive therapy in addition to usual erythromycin (and rifampin for certain difficult or refractory infections).

\section{Organisms in the Respiratory Flora Recently Recognized to Cause Infection}

Because present diagnostic tests cannot identify a causative organism for half the cases of CAP, possibly unrecognized microbes will be found to account for this void, as illustrated by the discovery of Legionella species. Equally plausible is that members of the normal microbial flora may become pathogenic when they are located in unusual portions of the respiratory tract or the immune status of the host is compromised. Cigarette smoking and related chronic bronchitis and COPD can favor respiratory colonization of S. pneumoniae and Hemophilus sp. [80] and increase the probability of infection with these bacteria. For example, $H$. influenzae was associated with tracheobronchitis and meningitis, but it was considered an unusual cause of pneumonia [49, 97] until more recently [64]. The same realization that $M$. (Branhamella) catarrhalis, considered to be a harmless part of the nasopharyngeal flora, could be a lower tract pathogen is rather recent [70].

Three other organisms have received recent attention: C. pneumoniae, C. pseudodiphtheriticum and Bordetella pertussis.

\section{Chlamydia}

Chlamydia consists of three species: C. psittaci, C. trachomatis, and C. pneumoniae, TWAR strain [38]. The last is associated with sinusitis, pharyngitis, bronchitis, and pneumonia and is now implicated in approximately $10 \%$ of outpatient and inpatient cases of pneumonia. The discovery and much of the substantive clinical research performed with this organism have been done by Grayston and colleagues in Seattle [36-38]. C. pneumoniae is given as the third or fourth most frequent cause of CAP [36], and indeed it was found as the etiology of 6-8\% of CAP cases in the three series we are monitoring, as tabulated by Marrie and referred to previously [54], ranking it approximately fourth. This newly identified species of Chlamydia is classified as a 
bacterium, but it only grows intracellularly. As $C$. pneumoniae can be difficult to grow in culture, identification of its specific DNA, using PCR amplification methods applied to throat swab material or respiratory secretions, has established its role in causing outbreaks of infections [37]. As with other acute respiratory infections, no complex of symptoms or findings is sufficiently characteristic of Chlamydia to permit a clinical diagnosis. Illness attributed to $C$. pneumoniae TWAR strain can be mild and relatively asymptomatic or can be in the form of bronchitis or pneumonia. However, the infection may be subacute, and recovery can be slow (lasting weeks or months) even with erythromycin or tetracycline treatment [36].

The frequency of persistent cough provides an interesting insight into the bacterium's pathogenesis and involvement with respiratory epithelial cells. Another strain, C. trachomatis, which is a cause of blinding trachoma and sexually transmitted infection, was investigated for its effect on ciliated nasal epithelial cells [50]. Recently, Avni-Shemer and Lieberman [8], using human ciliated bronchial epithelial cells obtained from nonsmoking adults at bronchoscopy, found that $C$. pneumoniae infection of cell cultures inhibited ciliary action within $48 \mathrm{~h}$. It is uncertain what caused the ciliostatic effect. For other bacteria, such as $P$. aeruginosa $[87,91,101]$ exotoxins and microbial products have been found to paralyze ciliary motion, perhaps creating the opportunity for microbes to bind to the cilia or adhere directly to the respiratory cell.

Persistent cough and development of hyperactive airway symptoms (asthma bronchitis) have been reported, posing an association between TWAR infections and asthma [36]. This is substantiated better now, based on detection of specific anti- $C$. pneumoniae IgE antibodies that developed in acute phase sera in $12 / 14$ children who presented with acute wheezing that was associated with culture-proven C. pneumoniae infection [23]. Therefore, this infection may cause its aftermath through effects on mucociliary function (prolonged cough) and by antigenic stimulation producing IgE antibody-induced asthma.

\section{Diphtheroids (Corynebacterium pseudodiphtheriticum, Prior Designation was $C$. hofmannii)}

Aerobic Gram-positive bacilli of Corynebacterium species are often part of the normal oropharyngeal microbial flora [51] but have been reported occasionally to cause various infections involving the respiratory and urinary tracts and endocarditis. These nondiphtheria corynbacteria are generally not considered seriously as human pathogens. However, this concept may need reevaluation because of two recent reports [1, 53], especially if diphtheroids are observed to be intracellularly located in PMN leukocytes in stained smears of good quality specimens of respiratory secretions. Among the 17 patients reported by Manzella and colleagues [53] from York Hospital in York, Pennsylvania, 12 were considered to have bronchitis and five pneumonia; most cases might be considered as a form of community-related infection as only four cases were designated as hospital acquired. Many of these patients were elderly (average age was about 68 years, range 40-93 years), and 14 had an underlying or multiple systemic conditions, such as congestive heart failure $(n=8)$, COPD $(n=8)$, cancer, renal failure, or diabetes; three deaths occurred [53]. Of two cases described in detail [53], 
one was of a fatal case with autopsy evidence of bilateral, acute bronchopneumonia; tissue staining showed PMN leukocyte inflammation containing diphtheroid-like Gram-positive rods and rare Gram-positive cocci in clusters. However, a culture of the lung specimen was not done. Among the 16 patients reported from Nagasaki University, Nagasaki, Japan [1], their demographic and underlying conditions were reasonably similar to the other series [53]. Both articles noted that fever was not always present and was found in $16 / 33$ patients from the combined series. Generally, the bacteria were sensitive to a $\beta$-lactam antibiotic or trimethoprim-sulfamethoxazole but could be resistant to erythromycin and clindamycin and borderline sensitive for quinoline antibiotics [1].

In summary, the isolation of diphtheroids from a properly cultured sputum specimen should not be dismissed as just usual respiratory flora and a contaminant in certain situations. A clue given from analysis of the Gram-stained smear is that nonpathogenic Corynebacterium species that are part of the normal oropharyngeal flora are not phagocytosed by PMN leukocytes; but if diphtheroids are intracellularly located, infection might receive greater consideration. Caution is required before declaring that these bacteria are a cause of pneumonia until more evidence of tissue infection (culturepositive evidence) is obtained, and more cases are reported. The need for strict criteria to determine causation was commented upon by both groups of authors $[1,53]$.

\section{Bordetella pertussis}

Until recently, this respiratory infection was considered controlled with infant and childhood immunization, and no longer was whooping cough a clinical consideration. However, vaccination programs are not provided as widely as needed, and immunity can wane in previously vaccinated persons, both contributing to a rise in cases of pertussis. For example, the incidence in New York state in 1993-1994 was the highest reported in 40 years with more than 400 cases; in 1975, there were less than 100 cases [34]. By comparison [34] for the United States nationwide, the Centers for Disease Control reported from 1993 6,586 cases of pertussis, whereas almost a decade before this number was 2,276 . Pertussis booster vaccine may be warranted for adults if this trend continues [34]. The disease is being recognized increasingly in adolescents and adults. In fact, adults may be a reservoir of the disease and have a role in its transmission [103]. As with C. pneumoniae, a persistent cough might alert one to the diagnosis of pertussis.

A study by Wright and colleagues [103] focused on persistent cough lasting 2 weeks or longer among adult patients evaluated in an emergency service and the prevalence of $B$. pertussis infection. Seventy-five adults, mean age 32 years \pm 10 years (S.D.), were analyzed on the basis of nasopharyngeal swabs for culture and direct fluorescent antibody assay and on acute and 1-month convalescent serology titers to pertussis (also Mycoplasma pneumoniae titers were measured). These were compared with control subjects without respiratory complaints. This study [103] revealed important findings: (1) all but one subject had complete vaccination during childhood; (2) none had a positive culture for $B$. pertussis, and only in one was a DFA test positive; and (3) 16 subjects (or 21\%) had a sufficiently high serologic titer on presentation or 
a rise found in the convalescent serum sample to qualify for the authors' diagnosis of recent infection. Thus, in adults with persistent cough, about $20 \%$ may have evidence of recent $B$. pertussis infection, certainly a large enough number to make this association one to consider in a differential diagnosis. As the patients have rather nonspecific symptoms, it would be difficult to distinguish them on clinical grounds [103]. This study and others referred to in the literature cited [103] would confirm the general impression that pertussis infection is on the rise.

\section{Hantavirus Pulmonary Syndrome}

To continue the motif of existing microbes causing serious infection when in different locations or circumstances, the respiratory tract has another recent example. The public is becoming accustomed, but undoubtedly is still worried, that killer forms of pneumonia continue to emerge; Legionella was reported only 20 years ago. Capturing the imagination most recently has been the mysterious Ebola virus infection apparently originating from Zaire in Central Africa but capable of spreading quickly or appearing as a subhuman primate infection. This was described in an enthralling book [76] and also presented with a slightly different scenario in a contemporary movie [21]. Prominent periodicals have devoted their covers and published articles depicting deadly microbes and dire consequences $[32,48]$. This is all superimposed on the fact that many other infections, heretofore seemingly contained by antibiotics, vaccination, or good hygienic practices, have returned; i.e. tuberculosis, cholera, typhus, and malaria are major concerns again [4]. The postantibiotic infectious era, as discussed [17], may be looming.

Joining this scene is the Hantavirus pulmonary syndrome [15, 20], (HPS) reported from the southwestern part of the United States in May 1993. During the Korean War, 45 years ago, illness with a related virus had been evaluated among United Nations troops, who developed fever, hemorrhagic signs, and acute renal failure. This illness was called Korean hemorrhagic fever. According to Dr. John White, ${ }^{3}$ it did not have an obvious respiratory component, and the Hantavirus strain Hantaan, which he and co-workers characterized [99], was one of several strains in the Bunyaviridae family of RNA viruses which could cause the hemorrhagic fever with renal syndrome. The virus infected rodents and was carried by them in the zoonotic phase of the infection. Dr. White said that investigators later were aware of the presence of a related virus in the United States from isolates in urban rats and feral rodents and that a hantavirus, later designated "Prospect Hill,' had been isolated in the area near Frederick, Maryland.

The outbreak of HPS [20] with related but unrecognized virus in the genus Hantavirus (family Bunyaviridae) $[15,69]$ heralded a much different problem with this type of infection: a fulminant pulmonary illness leading to an acute adult respiratory distress type syndrome and a high fatality rate; $13 / 17$ patients reported died. It was comforting to believe, if one was from the eastern United States, that this syndrome

\footnotetext{
${ }^{3}$ Personal comments from John D. White, Ph.D., Frederick, Maryland. Aside from his work in virology at the United States Army Research Institute of Infectious Diseases at Fort Detrick in Frederick, Maryland, Dr. White was the president of the American Lung Association, 1991-1992.
} 
might be localized elsewhere. However, about the same time as the original outbreak occurred in May-June 1993 [20], a 61-year-old male who was admitted to our medical center was found to have ARDS and subsequently HPS. The latter was discovered by serologic titers of Hantavirus antibodies, as reported by Hamory and colleagues [41]. This patient, who recovered, had been hiking along the Appalachian Trail from Georgia northward to Virginia, and he reported evidence of mice, rodent traps, and excreta in various shelters and bunkhouses along the way. This was the first reported case east of the Mississippi River and extended the range of this illness. At the time this report was published [41], the editorial note accompanying it mentioned that 98 cases had been identified in 21 states. This past spring (May 1995), a second person was reported to have died of HPS in New York state (Suffolk County). In this article [86], it was cited that nationwide there had been a total of 106 confirmed cases. Thus, the potential for this respiratory infection to occur is widespread and must be considered.

According to the definitive descriptions $[15,20]$, HPS may occur in reasonably healthy people and with a constellation of symptoms, usually including fever, myalgias, headache, cough, and nausea or vomiting. After a prodromal period of about 3-6 days of fever and myalgias (a flu-like beginning), progressive cough and shortness of breath can initiate a cardiopulmonary phase [15] that can be a prelude to progressive hypoxemia and acute respiratory failure. As there is no specific treatment, general support for ARDS, hypotension, and multiorgan failure is the best that can be offered. As described, mortality is high. Perhaps the most effective strategy is to take precautions not to create dust when in surroundings where rodent fecal matter is evident, as an infected aerosol is the route of lung inoculation. In this respect, Rosenthal [86] passed on helpful tips from New York State Health officials: prevent contact with rodent carcasses by using gloves when removing them from traps; do not stir up dust when cleaning areas containing rodent droppings; do not sweep or vacuum up droppings but douse them with water and bleach before removal.

In summary, the HPS is another example of a microbe, a genus of viruses found to infect rodents and previously known to cause a hemorrhagic renal failure syndrome, which now develops pathogenicity to involve the respiratory tract causing a fulminant, devastating pneumonia with high mortality. Younger, previously healthy persons seem likely to be frequent targets while pursuing outdoor activities given the nature of exposure to rodents and their excreta. Prevention in the form of care when cleaning areas likely to be contaminated with infected droppings is the best defense at present until a specific therapy is developed. It is encouraging [19] that immune animal and patient sera can cause cross-antibody neutralization of several strains of pathogenic Hantaviruses, opening the possibility for development of a vaccine and protective immunity.

Acknowledgments: I appreciate the review of the manuscript and helpful comments given by Michael S. Niederman, M.D., Director of Medicine and Respiratory Intensive Care Unit at the Winthrop University Hospital, Mineola, New York, and Joseph H. Bates, M.D., Chief, Medical Service, John L. McClellan Memorial Veterans Hospital and Professor and Vice Chairman, Department of Medicine, University of Arkansas for Medical Sciences, Little Rock. I also appreciate the secretarial assistance of Susan K. Crawford in the preparation of the manuscript. 


\section{References}

1. Ahmed K, Kawakami K, Watanabe K, et al. (1995) Corynebacterium pseudodiphtheriticum: a respiratory tract pathogen. Clin Infect Dis 20:41-46.

2. American College of Physicians (1982) Pneumococcal vaccine recommendation. Ann Intern Med 96:206-207

3. American College of Physicians (1994) Guide for Adult Immunization: Pneumococeal Infections, 3rd ed, Philadelphia, pp 107-114

4. Angier, N (24 August 1995) Deadly chess game: new clue to malaria. New York Times Service, International Herald Tribune, $\mathrm{p} 10$

5. Appelbaum PC, Bhamjee A, Scragg JN, et al. (1977) Streptococcus pneumoniae resistant to penicillin and chloramphenicol. Lancet 2:995-997

6. Austrian R (1977) Pneumococcal infection and pneumococcal vaccine. N Engl J Med 297:938-939

7. Austrian R, Gold J (1964) Pneumococcal bacteremia with especial reference to bacteremic pneumococcal pneumonia. Ann Intern Med 60:759-776

8. Avni-Shemer Y, Lieberman D (1995) Chlamydia pneumoniae-induced ciliostasis in ciliated bronchial epithelial cells. J Infect Dis 171:1274-1278

9. Bartlett JG, Mundy LM (1995) Community-acquired pneumonia: current concepts. N Engl J Med 333:1618-1624

10. Bates JH, Campbell GD, Barron AL, McCracken GA, et al. (1992) Microbial etiology of acute pneumonia in hospitalized patients. Chest 101:1005-1012

11. Bhardwaj N, Nash TW, Horwitz MA (1986) Interferon gamma activated human monocytes inhibit the intracellular multiplication of Legionella pneumophila. I Immunol 137:2662-2669

12. Broome CV, Facklam RR, Fraser DW (1980) Pneumococcal disease after pneumococcal vaccination. $\mathrm{N}$ Engl J Med 308:549-552

13. Bruyn GAW, Zegers BJM, van Furth R (1992) Mechanisms of host defense against infection with Streptococcus pneumoniae. Clin Infect Dis 14:251-262

14. Butler JC, Breiman RF, Campbell JF, et al. (1993) Pneumococcal polysaccharide vaccine efficacy. JAMA 270:1826-1831

15. Butler JC, Peters CJ (1994) Hantaviruses and Hantavirus pulmonary syndrome: state of the art clinical article. Clin Infect Dis 19:387-395

16. Byrd TF, Horwitz MA (1989) Interferon gamma-activated human monocytes downregulate transferrin receptors and inhibit the intracellular multiplication of Legionella pneumophila by limiting the availability of iron. J Clin Invest 83:1457-1465

17. Casadevall A, Scharff MD (1995) Return to the past: the case for antibody-based therapies in infectious diseases. Clin Infect Dis 21:150-161

18. C.D.C. reduces size of disease outbreak. (16 July 1995) Associated Press, Chambersburg, Pennsylvania, The New York Times

19. Chu Y-K, Jennings G, Schmaljohn A, et al. (1995) Cross-neutralization of Hantaviruses with immune sera from experimentally infected animals and from hemorrhagic fever with renal syndrome and Hantavirus pulmonary syndrome patients. J Infect Dis 172:1581-1584

20. Duchin JS, Koster FT, Peters CJ, et al. (1994) Hantavirus pulmonary syndrome: a clinical description of 17 patients with a newly recognized disease. N Engl J Med 330:949-955

21. Dworet L, Pool RR (1995) “Outbreak," director Wolfgang Peterson, producers Arnold Kopelson, Wolfgang Peterson, Gail Katz, starring Dustin Hoffman and Rene Russo, Warner Brothers

22. Edelstein PH (1993) Legionnaires' disease. Clin Infect Dis 16:741-749

23. Emre U, Sokolovskaya N, Roblin PM, et al. (1995) Detection of anti-Chlamydia pneumoniae IgE in children with reactive airway disease. J Infect Dis 172:265-267

24. Fang G-D, Fine M, Orloff J, et al. (1990) New and emerging etiologies for community-acquired pneumonia with implications for therapy. A prospective multi-center study of 359 cases. Medicine 69:307-316

25. Fang G-D, Yu VL, Vickers RM (1989) Disease due to the Legionellaceae (other than Legionella pneumophila). Medicine 68:116-132

26. Fein AM, Niederman MS (1995) Guidelines for the initial management of community-acquired pneumonia: savory recipe or cookbook for disaster. Am J Respir Crit Care Med 152:1149-1153 
27. Fick RB Jr, Reynolds HY (1983) Changing spectrum of pneumonia: news media creation or clinical reality? Am J Med 74:1-8

28. Finland M (1987) And the walls come tumbling down: more antibiotic resistance and now the pneumococcus. N Engl J Med 299:770-771

29. Fraser DW, Tsai TR, Orenstien W, et al. (1977) Legionnaires' disease: description of an epidemic of pneumonia. N Engl J Med 297:1189-1197

30. Friedland IR, McCracken GH Jr (1994) Management of infections caused by antibiotic-resistant Streptococcus pneumoniae. N Eng1 J Med 331:377-382

31. Gable CB, Holzer SS, Engelhart L, et al. (1990) Pneumococcal vaccine: efficacy and associated cost savings. JAMA 264:2910-2915

32. Gladwell M (17 and 24 July 1995) The plague year: the unscientific origins of our obsession with viruses. The New Republic, pp 38-46

33. Glick TH, Gregg MB, Berman B, et al. (1978) Pontiac Fever: an epidemic of unknown etiology in a health department. I. Clinical and epidemiologic aspects. Am J Epidemiol 107:149-160

34. Goldman EL (15 June 1995) New York records highest pertussis rate in 40 years. Intern Med News 28(12):7. Reporting the comments of Dr. Guthrie Birkhead, director of the Bureau of Communicable Disease Control, New York State Department of Health, Albany, from the annual meeting of new York State Academy of Family Physicians, Saratoga Springs, NY

35. Govaert ME, Thijs CTMCN, Masurel N, et al. (1994) The efficacy of influenza vaccination in elderly individuals. JAMA 272:1661-1665

36. Grayston JT (1992) Infections caused by Chlamydia pneumoniae strain TWAR. Clin Infect Dis 15: 757-763

37. Grayston JT, Aldous MB, Easton A, et al. (1993) Evidence that Chlamydia pneumoniae causes pneumonia and bronchitis. J Infect Dis 168:1231-1235

38. Grayston JT, Campbell LA, Kuo CC, et al. (1990) A new respiratory tract pathogen: Chlamydia pneumoniae strain TWAR. I Infect Dis 161:757-761

39. Gross PA, Hermogenes AW, Sacks HS, et al. (1995) The efficacy of influenza vaccine in elderly persons: a metaanalysis and review of the literature. Ann Intern Med 123:518-527

40. Hamill RJ, Musher DM, Groover JE, et al. (1992) IgG antibody reactive with five serotypes of Streptococcus pneumoniae in commercial intravenous immunoglobulin preparations. J Infect Dis 166: $38-42$

41. Hamory BH, Zwillich C, Bollard T, et al. (December 1994) Hantavirus pulmonary syndrome, Virginia, 1993. MMWR 43(47):876-877

42. Hansman D, Glasgow H, Sturt J, et al. (1971) Increased resistance to penicillin of pneumococci isolated from man. N Engl J Med 284:175-177

43. Hofmann J, Cetron MS, Farley MM, et al. (1995) The prevalence of drug-resistant Streptococcus pneumoniae in Atlanta. N Engl J Med 333:481-486

44. Horwitz MA, Silverstein SC (1981) Interaction of the Legionnaires' disease bacterium (Legionella pneumophila) with human phagocytes. I. L. pneumophila resists killing by polymorphonuclear leukocytes, antibody and complement. J Exp Med 153:386-397

45. Ingram JG, Plouffe JF (1994) Danger of sputum purulence screens in culture of Legionella species. J Clin Microbiol 32:209-210

46. Jacobs MR, Koornhof HJ, Robins-Browne RM, et al. (1978) Emergence of multiply resistant pneumococci N Engl J Med 299:735-740

47. 'Legionnaires' disease suspected in 45 patients (14 July, 1995). The Patriot (Harrisburg, Pennsylvania), The Patriot-News Company 154:167

48. Lemonick MD (12 September, 1994) Revenge of the killer microbes. Time Magazine, pp 62-69

49. Levin DC, Schwarz MI, Matthay RA, LaForce FM (1977) Bacteremic Hemophilus influenzae pneumonia in adults: a report of 24 cases and a review of the literature. Am J Med 62:219-224

50. Luzzatto E, Kopernik G, Sarov I, Priel Z (1989) Effects of Chlamydia trachomatis infection on ciliary activity in single cells from cultures of human nasal polyps. J Gen Microbiol 135:105-109

51. Mackowiak PA (1982) The normal microbial flora. N Engl J Med 307:83-93

52. Mandell LA (1995) Community-acquired pneumonia: etiology, epidemiology and treatment. Chest 108 (suppl.):35S-42S 
53. Manzella JP, Kellogg JA, Parsey KS (1995) Corynbacterium pseudodiphtheriticum: a respiratory tract pathogen in adults. Clin Infect Dis 20:37-40

54. Marrie TJ (1994) Community-acquired pneumonia: state of the art clinical article. Clin Infect Dis 18:501-515

55. Marrie TJ, Durant H, Yates L (1989) Community-acquired pneumonia requiring hospitalization: fiveyear prospective study. Rev Infect Dis 11:586-599

56. Martin RJ, Boguniewicz M, Henson JE, et al. (1993) The effects of inhaled interferon gamma in normal human airways. Am Rev Respir Dis 148:1677-1682

57. McDade JE, Shepard CC, Fraser DW, et al. (1977) Legionnaires' disease: isolation of a bacterium and demonstration of its role in other respiratory disease. N Engl J Med 297:1197-1203

58. Merrill CW, Gwaltney JM Jr, Hendley JO, Sande MA (1973) Rapid identification of pneumococci: Gram stain versus Quellung reaction. N Engl J Med 288:510-512

59. Muder RR, Yu VL, Fang G-D (1989) Community-acquired Legionnaires' disease. Semin Respir Infect 4:32-39

60. Mullooly JP, Bennett MD, Hornbrook MC, et al. (1994) Influenza vaccination programs for elderly persons: cost effectiveness in a health maintenance organization. Ann Intern Med 121:947-952

61. Mundy LM, Auwaerter PG, Oldach D, et al. (1995) Community-acquired pneumonia: impact of immune status. Am J Respir Crit Care Med 152:1309-1315

62. Munford RS, Murphy TV (1994) Antimicrobial resistance in Streptococcus pneumoniae: can immunization prevent its spread? J Invest Med 42:613-621

63. Musher DM (1992) Infections caused by Streptococcus pneumoniae: clinical spectrum, pathogenesis, immunity, and treatment. Clin Infect Dis 14:801-809

64. Musher DM, Kubitschek KR, Crennan J, Baughn RE (1983) Pneumonia and acute febrile tracheobronchitis due to Haemophilus influenzae. Ann Intern Med 99:440-450

65. Myerowitz RL, Pasculle AW, Dowling JN, et al. (1979) Opportunistic lung infection due to "Pittsburgh pneumonia agent." N Engl J Med 301:953-958

66. Naber SP (1994) Molecular pathology: diagnosis of infectious disease. N Eñng J Med 331:1212-1215

67. Nash TW, Libby DM, Horwitz MA (1984) Interaction between the Legionnaires' disease bacterium (Legionella pneumophila) and human alveolar macrophages. J Clin Infect 74:771-782

68. Nichol KL, Margolis KL, Wuorenma J, Von Sternberg, T (1994) The efficacy and cost effectiveness of vaccination against influenza among elderly persons living in the community. $N$ Engl $J$ Med 331:778-784

69. Nichol, ST, Spiropoulou CF, Morzunov S, et al. (1993) Genetic identification of a hantavirus associated with an outbreak of acute respiratory illness. Science 262:914-917

70. Nicotra B, Rivera M, Luman I, et al. (1986) Branhamella catarrhalis as a lower respiratory tract pathogen in patients with chronic lung disease. Arch Intern Med 146:890-893

71. Niederman MS (1993) Microbial flora of the respiratory tract: normal inhabitants and abnormal colonization. I. In: Bone R (ed) Infectious Diseases: General Considerations in Pulmonary and Critical Care Medicine. Mosby-Year Book, St. Louis, Vol 2 Part I, pp 1-12

72. Niederman MS, Bass JB, Campbell GD, et al. (1993) Guidelines for the initial management of adults with community-acquired pneumonia: diagnosis, assessment of severity, and initial antimicrobial therapy (ATS statement). Am Rev Respir Dis 148:1418-1426

73. Örtqvist A, Kalin M, Julander I, Mufson MA (1993) Deaths in bacteremic pneumococcal pneumonia: a comparison of two populations--Huntington, West Virginia, and Stockholm, Sweden. Chest 103: $710-716$

74. Plouffe JF (1995) Re-evaluation of the definition of Legionnaires' disease: use of the urinary antigen assay. Clin Infect Dis 20:1286-1291

75. Preston J (5 November, 1995) Fatal illness in Nicaragua is a mystery. New York Times

76. Preston R (1994) The Hot Zone, Random House, New York

77. Puren AJ, Feldman C, Savage N, et al. (1995) Patterns of cytokine expression in community-acquired pneumonia. Chest 107:1342-1349

78. Reynolds HY (1974) Prevention and future control of hospital-associated infections commonly caused by gram-negative bacteria. Prevent Med 3:507-514

79. Reynolds HY (1979) Legionnaires’ disease. In: Wyngaarden JB, Beeson PB, McDermott W (eds) Cecil's Textbook of Medicine, 15th ed. Saunders, Philadelphia, pp 359-360 
80. Reynolds HY (1987) Bacterial adherence to respiratory tract mucosa: dynamic interaction leading to colonization. Semin Respir Infect 2:8-19

81. Reynolds HY (1990) Community-acquired pneumonia: problems in respiratory care-current issues. In: (Witek TJ, Schachter EN (eds) Respiratory Public Health. Lippincott, Philadelphia, pp 20-30

82. Reynolds HY (1994) Host defense mechanisms in the respiratory tract. In: Stein's Textbook of Medicine, 4th ed. Mosby-Year Book, St. Louis, pp 1578-1585

83. Reynolds HY (1994) Cytokines: role in respiratory illnesses and potential control with immunomodulatory therapy. Focus and opinion: Intern Med 1(\#6):1-10

84. Robbins JB, Schneerson R, Szu SC (1995) Perspective. Hypothesis: serum IgG antibody is sufficient to confer protection against infectious diseases by inactivating the inoculum. J Infect Dis 171:13871398

85. Roig J, Domingo C, Morera J (1994) Legionnaires' disease. Chest 105:817-825

86. Rosenthal E (4 May, 1995) Second man dies from rare mouse-borne virus. The New York Times, p B5

87. Saiman L, Ishimoto K, Lory S, Prince A (1990) The effect of piliation and exoproduct expression on the adherence of $P$ seudomonas aeruginosa to respiratory epithelial monolayers. J Infect Dis 161:541548

88. Salo P, Örtqvist A, Leinonen M (1995) Diagnosis of bacteremic pneumococcal pneumonia by amplification of pneumolysin gene fragment in serum. J Infect Dis 171:479-482

89. Schluger NW, Rom WN (1995) The polymerase chain reaction in the diagnosis and evaluation of pulmonary infections: clinical commentary. Am J Respir Crit Care Med 152:11-16

90. Schwartz IS (1982) Pneumococcal vaccine: clinical efficacy and effectiveness. Ann Intern Med 96: 208-220

91. Seybold ZV, Abraham WM, Gazeroglu H, Wanner A (1992) Impairment of airway mucociliary transport by Pseudomonas aeruginosa products. Am Rev Respir Dis 146:1173-1176

92. Shapiro ED, Berg AT, Austrian R, et al. (1991) The protective efficacy of polyvalent pneumococcal polysaccharide vaccine. N Engl J Med 325:1453-1460

93. Simberkoff MS, Cross AP, Al-Ibrahim M, et al. (1986) Efficacy of pneumococcal vaccine in high risk patients: results of a Veterans Administrative cooperative study. N Engl J Med 315:1318-1327

94. Skerrett SJ (1994) Legionnaires' disease: mediguide to Pulm Infect Dis 1:1-7

95. Skerrett SJ, Martin TR (1994) Intratracheal interferon-gamma augments pulmonary defenses in experimental legionellosis. Am J Respir Crit Care Med 149:50-58

96. Task Force on Adult Immunization (members representing the American College of Physicians, Infectious Diseases Society of American and Centers for Disease Control and Prevention) (1994) Adult immunization 1994. Ann Intern Med 121:540-541

97. Tillotson JR, Lerner AM (1968) Hemophilus influenzae bronchopneumonia in adults. Arch Intern Med 121:428-432

98. Tuomanen EI, Austrian R, Masure HR (1995) Pathogenesis of pneumococcal infection. N Engl J Med 332:1280-1284

99. White JD, Shirey FG, French GR, et al. (1982) Hantaan virus, aetiological agent of Korean hemorrhagic fever, has Bunyaviridae-like morphology. Lancet 1:768-771

100. Willems IS, Sanders CR, Riddiough MA, Bell JC (1980) Cost effectiveness of vaccination against pneumococcal pneumonia. N Engl J Med 303:553-559

101. Wilson R, Pitt T, Taylor G, et al. (1987) Pyocyanin and 1-hydroxyphenazine produced by Pseudomonas aeruginosa inhibit the beating of human respiratory cilia in vitro. J Clin Invest 79:221-229

102. Woo AH, Yu VL, Goetz A (1986) Potential in-hospital modes of transmission of Legionella pneumophila. Am J Med 80:567-573

103. Wright SW, Edwards KM, Decker MD, Zeldin MH (1995) Pertussis infection in adults with persistent cough. JAMA 273:1044-1046

Accepted for publication: 2 January 1996 